lamotrigine was superior to placebo. (Chronicle E, Mulleners W. Cochrane Database Syst Rev 2004;(3):CD003226).

\title{
ACUTE HEADACHE AND SICKLE CELL DISEASE
}

Researchers at Children's Hospital of Philadelphia and Children's Hospital of Michigan, Detroit, determined the frequency of acute care visits for headache in children with homozygous sickle cell disease (SCD-SS), neuroimaging studies, and associated CNS events. Of 2685 acute care visits by children with SCD-SS, 102 (3.8\%) presented with headache as the chief complaint, and acute CNS events were diagnosed at $6.9 \%$ of these visits. Neuroimaging, performed in $42.2 \%$ of visits, identified acute CNS events in $16.3 \%$ of studies. Risk factors for acute CNS events that warrant confirmatory neuroimaging include older age, history of stroke, transient ischemic attack, or seizure, neurologic symptoms, focal neurologic exam, and elevated platelets. (Hines PC, McKnight TP, Seto W, Kwiatkowski JL. Central nervous system events in children with sickle cell disease presenting acutely with headache. J Pediatr September 2011;159:472478). (Reprints: Dr Janet L Kwiatkowski MD, Children's Hospital of Philadelphia, 34th Street, Colkett Bldg, Room 11024, Hematology, Philadelphia, PA 19104. E-mail: Kwiatkowski@email.chop.edu.

COMMENT. The authors recommend neuroimaging in children with SCD-SS who present with acute headache and have associated neurologic symptoms, focal findings on exam and /or a history of stroke, transient ischemic attack, or seizure, or elevated platelet count. Consider imaging in patients with atypical headache, abnormal transcranial Doppler, vasculopathy on MRA, and/or silent infarcts.

\section{AUTONOMIC HEADACHE}

The features of headache attributed to autonomic dysreflexia after spinal cord injury (SCI) were determined by a literature search at the University of Toronto, Canada. Presenting features were sudden onset, severe throbbing headache, accompanied by increased blood pressure, altered heart rate, cutaneous vasodilation, hyperhidrosis and flushing cranial to the level of SCI, blurred vision, syncope, and anxiety. Triggers of the headache include bladder distension, constipation, sunburn, menstruation, and pulmonary infarct. (Furlan JC. Headache attributed to autonomic dysreflexia. An underrecognized clinical entity. Neurology Aug 23, 2011;77:792-798). (Respond and reprints: Dr Julio C Furlan, Toronto Western Research Institute, 399 Bathurst Street, Toronto, Ontario, Canada M5T 258. E-mail: jcfurlan@gmail.com).

COMMENT. Autonomic dysreflexia after SCI (at T6 or above) is characterized by initial low blood pressure and bradycardia, followed by volatile and episodic extreme hypertension, accompanied by headache and upper body flushing provoked by triggers. A triad of cephalalgia, hyperhidrosis, and cutaneous vasodilation occurs in $85 \%$ cases. The prevalence of SCI in children is $2.4 /$ million and $25 /$ million in adolescents, mainly car and motorcycle accidents. (Hagen EM et al. Spinal cord 2011; May 10. Epub ahead of print). 